\title{
Estrategias de gestión de la información para cubrir necesidades laborales especializadas
}

\section{Administrative strategies to meet the needs of specialized occupations}

\author{
Dr. Joan Francesc Fondevila Gascón \\ Director, Departamento de Ciencias de la Comunicación, \\ Universitat Abat Oliba CEU (UAO-CEU) \\ Sergio Estella García \\ Profesor, Universitad Francisco de Vitoria (UFV) \\ Dr. Josep Lluís del Olmo Arriaga \\ Profesor, Universitat Abat Oliba CEU (UAO-CEU)
}

Fecha de recepción: 16 de septiembre de 2012

Fecha de revisión: 26 de diciembre de 2012

Para citar este artículo: Fondevila Gascón, J.F.; Estella García, S. y del Olmo Arriaga, J.L. (2013): Estrategias de gestión de la información para cubrir necesidades laborales especializadas, Icono 14, volumen 11 (1), pp. 217-231. doi: 10.7195/ri14.v11i1.511 


\section{Resumen}

La cobertura de necesidades laborales especializadas en el entorno digital requiere de nuevas estrategias, adaptadas a las peculiaridades del entorno virtual. En este artículo, que sigue la metodología del estudio de caso, planteamos el uso de redes sociales para cubrir una necesidad muy específica en el entorno empresarial. De esta manera estudiamos la repercusión de un suceso, que parte de un mensaje, se multiplica y alcanza a miles de receptores, para concluir de forma eficaz. La correcta gestión de los canales y de la difusión de la información garantiza un uso correcto del medio.

\section{Palabras clave}

Información - red social - Internet - empleo - estrategia

\section{Abstract}

Coverage of specialized labor needs in the digital environment requires new strategies, adapted to the peculiarities of the virtual environment. In this article, which follows the case study methodology, we propose the use of social networks to cover a very specific need in the business environment. Thus we study the impact of an event, that part of a message, multiply and reach thousands of recipients, to complete effectively. Proper channel management and dissemination of information ensures proper use of the medium.

\section{Key Words}

Information - social network - Internet - employment - strategy 


\section{Introducción. Las estrategias de gestión de la}

\section{información en redes sociales}

Las redes sociales constituyen un fenómeno creciente, casi exponencial, que está provocando un cambio de paradigma en las estrategias de gestión empresarial. El aumento de usuarios de social media (HighBeam Research, 2011) ha generado áreas de análisis especializadas, cuyo recorrido científico es aún modesto a raíz de la incipiencia del fenómeno y de la carencia de una perspectiva longitudinal.

En el ámbito de la comunicación y el marketing, se están desarrollando estrategias de Social Engine Optimization (SE0), Social Engine Marketing (SEM) y Social Media Marketing (SMM). En el entorno de gestión de la información para cubrir necesidades laborales especializadas, la literatura científica rastreada es mínima 0 inexistente. Se han estudiado los esquemas de trabajo en red y la identidad social de profesionales red y profesionales no red pertenecientes a una o varias organizaciones simultáneamente (Moreno, 2010), y se determina el fortalecimiento de las identidades sociales complementarias, con el consiguiente riesgo de debilitamiento de la identidad social principal. También se han estudiado los factores que favorecen que un contacto se convierta en fuente de información en una red social en línea: conocimiento sobre el contacto, conocimiento de lo que el contacto sabe, cercanía social, cierto prestigio del contacto, conocimiento del contacto en persona y accesibilidad (Ureña y Valenzuela, 2011).

Desde la perspectiva del profesional de la información y la comunicación, es necesario disponer de herramientas metodológicas suficientemente sólidas como para llevar a cabo una acción de propuesta laboral en redes sociales, cuyas ramificaciones en movilidad son elevadas (Zenithoptimedia, 2010).

En el engarce entre estrategias de recruitment e Internet, los social media se están erigiendo en epicentro de interés científico. El estudio sobre las redes sociales es reciente y sin perspectiva longitudinal. Podemos definir una red social como una suma de servicios en Internet que permite a un usuario crear un perfil público o semipúblico dentro de una red o sistema, compartir contacto con otros usuarios y visualizar y rastrear esa lista de contactos y la de otros usuarios dentro esa red. La denominación de las conexiones acostumbra a diferir de una red social a otra (Boyd y Ellison, 2007).

DOI: ri14.v11i1.511 | ISSN: 1697-8293 | Año 2013 Volumen 11 Nº1 | ICONO14 
Una red social en Internet, un sistema social virtual, requieren en la base un establecimiento claro del concepto de perfil. El mecanismo habitual es que el usuario aporta la información y los datos personales que desea, siguiendo el estilo de un formulario. Cada miembro de la red sigue sus propias estrategias, en función de si su misión consiste en conseguir muchas amistades de primer, segundo o los niveles que sean necesarios. En función del grado de profundidad y de la amplitud, la red genera usuarios siguiendo métricas diversas, de las que se abastecen las bases de datos cruzadas de e-commerce (CMT, 2012) y de los que también pueden obtener rendimiento los medios de comunicación digitales (Fondevila, Del Olmo, Sierra, 2011).

Las métricas que se pueden tener en cuenta son diversas, y su uso aún se halla en fase primigenia en la mayor parte de casos. La centralidad es el poder social de un nodo en función de lo bien que se conecte éste a la red. Se mide por la intermediación, la cercanía y el grado. El flujo de centralidad de intermediación es el grado en que un nodo contribuye a la suma del flujo máximo entre todos los pares de nodos, excluyendo ese nodo. La cercanía es el grado en que un usuario está cerca de todos las demás en una red. De ahí brota el coeficiente de agrupamiento (la probabilidad de que dos personas vinculadas a un nodo se asocien a sí mismas). El grado es el recuento del número de vínculos con otros usuarios en la red.

Se han llevado a cabo propuesta de taxonomía de las redes sociales. Así, existen redes sociales directas e indirectas. Las directas son aquellas en las que prima la horizontalidad y la igualdad de condiciones en la información compartida. En las indirectas, un usuario controla el debate y no necesariamente los perfiles son públicos (foros y blogs). Las redes directas pueden ser, según su finalidad, de ocio o de uso profesional, cercanas al objetivo del recruitment. Según el modo de funcionamiento, puede haber redes de contenidos, basadas en perfiles (personales o profesionales) y de microblogging. En función del grado de apertura, las redes abiertas pueden ser públicas o privadas. Según el nivel de integración, las redes son horizontales o verticales (ONTSI, 2012).

El concepto de interactividad entre usuarios nos lleva a diferentes tipos de redes sociales según las relaciones sociales que se establecen en ellas. Así, las redes pueden ser dirigidas (fan pages de Facebook, por ejemplo), es decir, dotadas de relación social no bidireccional, o no dirigidas o recíprocas (amistades de Facebook). También pueden ser explícitas (en ellas los usuarios declaran la relación: es el caso

ICONO14 | Año 2013 Volumen 11 № 01 | ISSN: 1697-8293 | DOI: ri14.v1 1i1.511 
de una amistad de Facebook) o implícitas (la relación depende del comportamiento, como ocurre con los comentarios en foros). La acción natural de los usuarios es establecer contactos, sinónimo de más popularidad y más posibilidades de interacción. Una cifra significativa es la media de contactos, que en redes sociales es de 195 (InSites Consulting, 2010). La interactividad puede ser diferente o similar a la presencial. Así, encontramos la comunicación mediada por ordenador (Wellman, 2004), el anonimato del Internet Relay Chat (IRC), el Bulletin Board System (BBS) o la ruptura con las estructuras de conformación identitaria (Johnson y Miller, 1998), lo que puede conducir a un laboratorio de la identidad (Turkle, 1995) o a los flames.

\section{Material y métodos: aplicación de la}

\section{metodología para cubrir necesidades laborales}

\section{especializadas utilizando redes sociales}

En la investigación partimos de la base que la comunicación está sufriendo una división y un cambio de paradigma: de vertical a horizontal. Emisor y receptor pueden emitir juicios, intercambiar opiniones y relacionarse, lo que se ha analizado en entornos como el académico (Cladellas, Cárcamo y Castelló, 2011). La información está sufriendo un proceso de desintermediación, controvertido (Fourie, 2001). Los usuarios eligen y pueden convertirse en personajes influyentes, sin necesidad de grandes grupos portavoces de información sesgada. Las opiniones se forman de manera disgregada y se agrupan -cuando lo hacen- en grupos con intereses comunes.

Pese al exceso de información disponible en Internet, la posible desinformación y la dificultad de procesado y selección, se pretende el establecimiento de mecanismos que permitan la transformación en sabiduría (DIKW: Data, Information, Knowledge y Wisdom). El objetivo consiste en utilizar redes sociales para cubrir una necesidad muy específica en el entorno empresarial. La hipótesis de nuestra investigación es que un uso racional y especializado de redes sociales para conseguir cubrir un puesto de trabajo especializado conduce a aspirantes muy adecuados 
a los parámetros de la demanda.

La metodología es el estudio de caso y el experimento se ha llevado a cabo en la empresa Simbiótica (dedicada a diseño y estrategia digital en los ámbitos de branding y marketing online), en Madrid, en junio de 2011. Esa empresa precisa de diseñadores de interfaces gráficas y experiencias memorables para las marcas, y de perfiles técnicos con un profundo conocimiento de lenguajes de programación apropiados, para llevar a cabo tales proyectos en Internet.

\section{Resultados}

El 8 de junio de 2011 Simbiótica publicó una oferta de trabajo en busca de un programador, con conocimientos de los lenguajes de programación open source, con los que habitualmente trabajan, PHP, MySQL, en entorno Apache, y que tuviera interés en seguir aprendiendo, ya que el entorno de trabajo y el medio en sí lo requieren. Previamente, la publicación de ofertas había desembocado en perfiles con relación muy tangencial a la demanda de empleo. El reto consistía en presentar una oferta de trabajo especializada, que permitiera una criba rápida y evitara las ineficiencias ligadas a la recepción de un volumen ingente de curriculum vitae (CV). El resultado de la estrategia sería poder procesar correctamente los CV recibidos.

La estrategia para cubrir la demanda de empleo consistía en diferenciarse de otras ofertas. Se siguieron los siguientes pasos:

a) Publicación. Dado que el sector de la ingeniería informática (en particular, los programadores en Internet) está muy solicitado, se escribió una oferta de trabajo cuya intención era diferenciarse, ya que su redacción se realizó en pseudocódigo a fin de ser accesible únicamente para usuarios con conocimientos de lenguajes de programación (Figura 1). Se trataba de una vía innovadora y segmentadora de presentar la oferta, con el fin de evitar candidatos que no se ajustasen al perfil. La oferta recogía ubicación de la empresa, descripción del perfil, disponibilidad de horario, contacto, requerimientos y opciones negociables, los mismos ingredientes que en otras ofertas de trabajo, pero condimentados de manera alternativa. 


\section{Figura 1. Oferta especializada lanzada en Twitter}

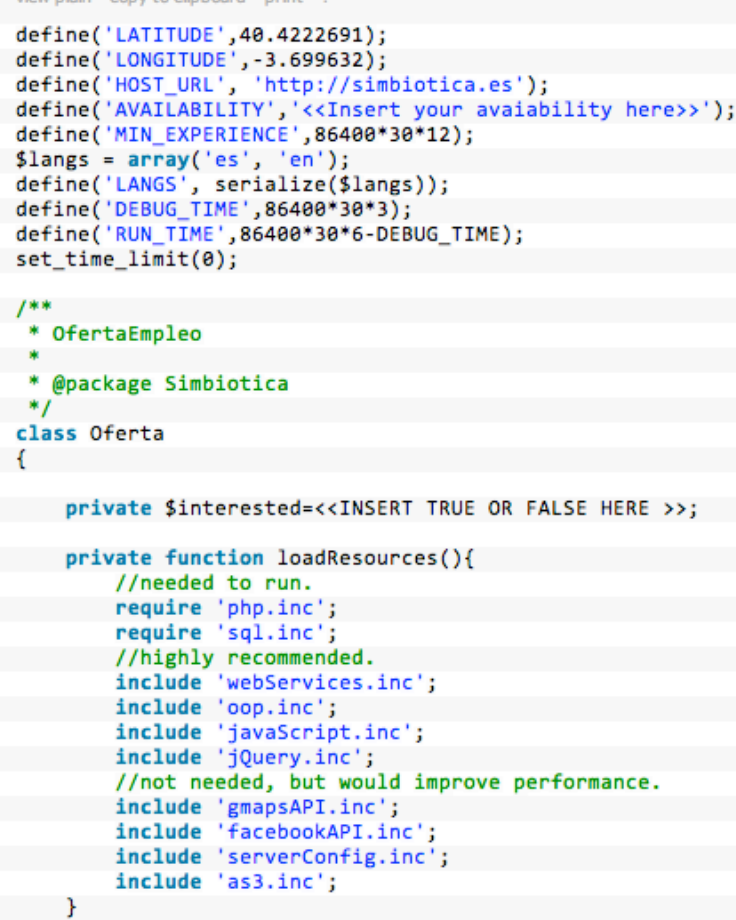

Fuente: Página de Internet (simbiotica.es/oferta)

b) Divulgación en una red social. Como segundo paso, se difundió la oferta, mediante algo tan sencillo como un tweet y el apoyo de algunos seguidores de Twitter (red de microblogging, 140 caracteres) de @simbioticos. En pocos minutos, el tweet había llegado a personas influyentes, que lo retuiteaban. A su vez sus, followers retuiteaban también. En poco tiempo, la recientemente creada cuenta de Twitter de la empresa, con algo más de 35 seguidores, alcanzaba los 500 retuits. La llegada del tweet a algunos usuarios influyentes (nodo importante) en la comunidad de programadores open source fue suficiente para conseguir una gran visibilidad.

El tweet contenía el siguiente call to action: "Buscamos back-end developer: http://simbiotica.es/oferta/ Se agradecen los retweets".

c) Impactos en redes sociales. Como tercera fase, y adicionalmente a los mencionados 500 retweets, se consiguieron otros logros para la empresa (Tablas 1 y 2):

- la oferta fue portada de meneame.es: más de 500 meneos y más de 100 comentarios, y fue la noticia más comentada durante 48 horas; 
- portada de microsiervos.com, lo que ayudó a aumentar la visibilidad de la oferta;

- 37.545 visitas a la oferta el primer día y cerca de 100.000 en una semana (datos recopilados por Google Analytics);

- menciones en miles de páginas y repercusión mundial de la oferta;

- aumento del prestigio de la empresa;

- nuevos followers en el Twitter de la empresa;

- 300 visitas diarias de media en la página los siguientes 4 meses (mientras que la media inicial estaba en 50 visitas diarias antes de publicar la oferta);

- contactos con otras empresas interesadas del sector;

- repercusión entre los clientes de la empresa;

- filtraje del volumen de CV recibidos;

- $\mathrm{y}$, last but not least, un programador en plantilla.

Tabla n¹. Número de visitas a la oferta durante las 24 primeras horas (8 de junio de 2011)

\begin{tabular}{|l|l|l|l|}
\hline Hora de visita & $\mathbf{N}^{0}$ de visitas & Hora de visita & $\mathbf{N}^{\mathbf{0}}$ de visitas \\
\hline $08: 00$ & 3 & $20: 00$ & 1559 \\
\hline $09: 00$ & 22 & $21: 00$ & 1374 \\
\hline $10: 00$ & 364 & $22: 00$ & 1535 \\
\hline $11: 00$ & 896 & $23: 00$ & 1273 \\
\hline $12: 00$ & 1019 & $00: 00$ & 802 \\
\hline $13: 00$ & 10150 & $01: 00$ & 474 \\
\hline $14: 00$ & 5317 & $02: 00$ & 250 \\
\hline $15: 00$ & 3823 & $03: 00$ & 177 \\
\hline $16: 00$ & 4097 & $04: 00$ & 185 \\
\hline $17: 00$ & 2360 & $05: 00$ & 157 \\
\hline $18: 00$ & 1734 & $06: 00$ & 127 \\
\hline $19: 00$ & 2003 & $07: 00$ & 229 \\
\hline
\end{tabular}

Fuente: Elaboración propia. 
Tabla n². Tráfico directo/visitantes exclusivos por país/territorio (día previo a su publicación, día de la oferta y día posterior)

\begin{tabular}{|l|l|l|l|}
\hline PAÍs & $\begin{array}{c}\text { 7 JUNI0 2011 } \\
\text { (290 visitantes } \\
\text { exclusivos) }\end{array}$ & $\begin{array}{c}\text { 8 JUNI0 2011 } \\
(36.368 \text { visitantes } \\
\text { exclusivos })\end{array}$ & $\begin{array}{c}\text { 9 JUNI0 2011 } \\
\text { 9.463 visitantes } \\
\text { exclusivos }\end{array}$ \\
\hline Argentina & 2 & 180 & 189 \\
\hline Chile & 1 & 93 & 34 \\
\hline Colombia & 2 & 86 & 29 \\
\hline República Checa & 0 & 515 & 51 \\
\hline Alemania & 3 & 94 & 27 \\
\hline España & 145 & 7625 & 2196 \\
\hline Francia & 2 & 75 & 97 \\
\hline Reino Unido & 7 & 280 & 57 \\
\hline Irlanda & 1 & 30 & 2 \\
\hline India & 1 & 5 & 1 \\
\hline Italia & 0 & 39 & 8 \\
\hline Japón & 1 & 8 & 3 \\
\hline Canadá & 0 & 0 & 28 \\
\hline
\end{tabular}

Fuente: Elaboración propia a partir de datos de www.google.com/analytics

\section{Discusión}

La estrategia de oferta de empleo en Internet arrojó resultados sorprendentes, vinculados a la exponencialidad (Gilder, 2002) inherente al ecosistema virtual. La naturaleza de la red obliga a realizar una serie de cambios en los paradigmas de la comunicación: se requiere un flujo de contenidos constante, el denominado cloud journalism (Fondevila, 2010b), y se produce un estructura horizontal y accesible de las periferias al centro y viceversa.

Uno de los obstáculos es la carencia de universalidad en el acceso a la red, aunque este factor apenas afectó a la campaña, ya que se dirigía a un perfil especializado y usuario natural de Internet. Desde 1984, con la aparición del MAC, se fomenta la autoedición. Las cámaras fotográficas e incluso los móviles graban vídeo a resoluciones elevadas, lo que permite reducir los costes para realizar una película, un spot o un documental. Eso va en la línea del periodismo ciudadano 
(Gillmor, 2004), que permite generar contenidos, aunque sean una mera fuente.

Las redes sociales están impulsando cambios en la relación entre los individuos y las grandes organizaciones y corporaciones a raíz de que la comunicación ya no es unidireccional. Las jerarquías se desvanecen. Los estudios clásicos sobre relación entre medios de comunicación y poder (McQuail, 1994) no contemplaban Internet, ni otro medio que se pudiera asemejar. En ninguno de los casos se planteaba un modelo mediado por el propio público. Internet, las redes sociales y la web sociosemántica (Morato, Fraga y Moreno-Pelayo, 2008) han roto esa barrera. Estos nuevos modelos se ajustan en mayor medida al modelo plural, no al modelo dominante. La audiencia es selectiva, fragmentada y activa, lo que permite obtener puntos de vista variados y enriquecidos.

Para una correcta estrategia de lanzamiento de una oferta laboral hay que centrarse en la relevancia de la información per se. El gran volumen de información inherente a Internet y a otros ámbitos puede originar desinformación (De Pablos, 2008). El ritmo de procesamiento de información es cada vez más exigente. En el nuevo ecosistema, se erigen nuevos tipos de liderazgo basados en la confianza en otros usuarios que proveen información de calidad: ello propicia la desintermediación.

Los usuarios son los que acreditan la validez de la información, que circula por nuevos canales. La transtemporalidad y la transespacialidad de Internet rompen los esquemas habituales, así como la interactividad (Fondevila y Del Olmo, 2011), el multimedia y el hipertexto (Fondevila, 2010a). El usuario elige de dónde recibe la información, la contrasta, la selecciona, la procesa, la interioriza y puede proyectar sus interpretaciones. Y eso de traslada a una sencilla oferta de empleo.

Sin embargo, Internet, pese a su relativa juventud, no se encuentra libre de monopolios. Algunos grupos multimedia (Arsenault y Castells, 2008) que surgieron con otras vocaciones abren sus puertas a otros medios tradicionales con gran poder en otros entornos y están copando algunos de los negocios. Algunos over the top y empresas del sector tecnológico (Apple, Google, Yahoo!, Amazon, Facebook, Twitter, LinkedIn, Pinterest) comienzan a monopolizar algunos contenidos del mercado (Fondevila, 2008). De hecho, se abastecen de los contenidos generados gratuitamente por los usuarios para pertrechar una masa crítica que les aporta ingresos por vía publicitaria o incluso del mercado de valores. La diferencia es que ahora es más sencillo encontrar fuentes alternativas.

ICONO14 | Año 2013 Volumen 11 № 01 | ISSN: 1697-8293 | DOI: ri14.v11i1.511 
Una de las cuestiones fundamentales para la propagación de la información en cualquier red es el nodo. En Internet, como en cualquier otro medio, la importancia de un nodo es indiscutible; de hecho, se convierte en uno de los principales pilares para la difusión en diversos círculos, ya que aligera la velocidad de transmisión. En el caso de la oferta de Simbiótica, unos de los factores concluyentes para que se propagase al ritmo al que lo hizo fue la conexión encontrada entre la empresa y algún gurú de programación open source, que facilitó la rapidez e hizo de nexo entre la empresa y otros nodos, conectando la oferta con otros gurús de programación. Así se catapultó en muy pocos pasos y se transformó de evento local a global en pocas horas.

La identidad humana de los usuarios, asociada a la digital, transmite confianza en redes sociales como Twitter, que destierra las identidades anónimas. Esa confianza conduce a retuitear. La protección de la identidad, la posibilidad de engaño o la invención de perfiles es una cuestión a debate. Así, una estrategia boicoteadora de la competencia puede hundir una compaña de comunicación. Por contra, los medios sociales pueden reafirmar colectivos (Kayani, 1998) o crear relaciones nuevas (Smith y Kollock, 1999).

Las redes sociales crean ecosistemas civilizados y gestionados por los propios usuarios, que pueden llegar a autorregularse, en una especie de homeostasis. Un evento como una oferta de trabajo supone un caso de civilización del ciberespacio (Sterling, 1993). Una oferta se propagó dentro de las redes gracias al hecho que se comprendía lo que se buscaba con ese discurso (líneas de código). Las más de 37.500 visitas el primer día (Tabla 3) así lo demuestran. La cantidad de CV recibidos fue comedida: el nivel de civilización de los usuarios ayudó a que la oferta no supusiera ningún tipo de caos para la empresa.

La estrategia de gestión de información condujo a una selección natural llevada a cabo por usuarios que conocían el medio, el Twitter de Simbiótica. Para que una comunicación sea eficaz no siempre son necesarias grandes bases de datos (big data). En algunos sectores prima la calidad. A partir de un número de seguidores bastante reducido (Simbiótica no alcanzaba los 40 seguidores), se consiguieron una difusión y una repercusión muy elevadas. Gracias a la inteligencia de los usuarios la información se difundió correctamente, esto es, en los círculos apropiados de un público segmentado que comprendía la oferta. Por tanto, nos encontramos ante una oferta de nicho, que no hubiera podido conseguir esa distribución selec- 
tiva sin Internet.

\section{Tabla $n^{03}$. Visitantes exclusivos en los meses previos y meses posteriores a la publicación de la oferta (abril 2011-abril 2012)}

\begin{tabular}{|l|l|}
\hline MES & VISITAS EXCLUSIVAS \\
\hline Abril 2011 & 115 \\
\hline Mayo 2011 & 78 \\
\hline Junio 2011 & 52.144 \\
\hline Julio 2011 & 700 \\
\hline Agosto 2011 & 419 \\
\hline Septiembre 2011 & 708 \\
\hline Octubre 2011 & 397 \\
\hline Noviembre 2011 & 565 \\
\hline Diciembre 2011 & 209 \\
\hline Enero 2012 & 285 \\
\hline Febrero 2012 & 276 \\
\hline Marzo 2012 & 319 \\
\hline Abril 2012 & 267 \\
\hline
\end{tabular}

Fuente: Elaboración propia a partir de datos de www.google.com/analytics

Así pues, hallamos una audiencia masiva pero no de masas. Esa es una clave en Internet. Se salta de lo público-lugar a lo público-espacio. El caso que hemos analizado permite identificar a Internet como un aliado para realizar cierto tipo de comunicaciones, destinadas al público adecuado en el entorno adecuado, sin necesidad de lanzar mensajes indiscriminados.

La velocidad de propagación es otra de las ventajas del círculo virtual aquí expuesto. Incluso teniendo en cuenta que la oferta no sólo se lanzó en castellano (tercer idioma más utilizado en Internet) -ya que, aunque el tweet estaba en castellano, la oferta propiamente se escribió en inglés (lengua utilizada por convenio en todos los lenguajes de programación)-, el evento traspasó las fronteras locales en un corto plazo de horas, siendo consultada en todos los continentes y en más de treinta lenguas del mundo.

Del estudio llevado a cabo se infiere, por tanto, que las redes sociales pueden permitir la cobertura de necesidades laborales especializadas. A partir de un caso 
particular de divulgación de una oferta de empleo especializada, combinando en Twitter lenguaje convencional y código para segmentar la audiencia, observamos un resultado marcado por la eficiencia y la elevada repercusión de un evento. Igualmente, la gestión de los canales virtuales y de la difusión de la información conduce a resultados extrapolables a otras empresas y actividades.

\section{Referencias}

Arsenault, A. H. \& Castells, M. (2008). The structure and dynamics of global multimedia business networks. International Journal of Communication, 2(1), 707-748.

Boyd, D. \& Ellison, N. (2007), “Social Network Sites: Definition, History and Scholarship", Journal of Computer-Mediated Communication.

Cladellas, R., Cárcamo, L. R. \& Castelló, A. (2011). Motivación y estimación del tiempo en el uso de herramientas internet informacionales y dialógicas. $E l$ profesional de la información, 20(1), 25-31.

CMT (Comisión del Mercado de las Telecomunicaciones) (2012). Informe e-commerce. Barcelona: CMT.

De Pablos Coello, J. M. (2008). El frenesí comunicativo como desinformación. Comunicar: Revista científica iberoamericana de comunicación y educación, 31, 173-179

Fondevila Gascón, J. F. (2008). La alianza entre el periodismo digital y tradicional y los operadores de telecomunicación: hacia un rendimiento óptimo de la red. Barcelona: II Congreso Nacional Ulepicc-España.

Fondevila Gascón, J. F. (2010a). Multimedia, digital press and journalistic genres in Catalonia and in Spain: an empirical analysis. Communication Studies Journal, 7, 81-95.

Fondevila Gascón, J. F. (2010b). El cloud journalism: un nuevo concepto de producción para el periodismo del siglo XXI. Observatorio (OBS*) Journal, 4(1), 19-35.

Fondevila Gascón, J. F. \& Del Olmo Arriaga, J. L. (2011). La interactividad y el multimedia en la prensa digital internacional: los casos de España y el Reino Unido. Bilbao: III Congreso Internacional de Ciberperiodismo y Web 2.0 “ $\mathrm{La}$ 
transformación del espacio mediático", Facultad de Ciencias Sociales y de la Comunicación de la Universidad del País Vasco.

Fondevila Gascón, J. F., Del Olmo Arriaga, J. L. \& Sierra Sánchez, J. (2011). New communicative markets, new business models in the digital press. Tripodos (Extra 2011-VI International Conference on Communication and Reality-Life without Media, Universitat Ramon Llull), 301-310.

Fourie, I. (2001). ¿Debemos tomarnos en serio la desintermediación? Anales de documentación: Revista de biblioteconomía y documentación, 4, 267-282.

Gilder, G. (2002). Telecosm: The World After Bandwidth Abundance. New York: Touchstone.

Gillmor, D. (2004). We the Media: Grassroots Journalism by the People, for the People. Sebastopol: 0'Reilly Media.

Highbeam Research (2011). Social Media Popularity Report. Chicago: HighBeam Research.

Insites Consulting (2010). Social Media around the world. London: InSites. Johnson, D. \& Miller, K. (1998). Anonymity, pseudonymity, or inescapable identity on the net. Computers and Society, 28(2). New York: ACM.

Kayany, J. M. (1998). Contexts of uninhibited online behaviour: Flaming in social newsgroups on usenet. Journal of the American Society for Information Science, 49(12), 1135-1141.

McQuail, D. (1994). Mass Communication Theory: An Introduction. London: SAGE Publications Ltd.

Morato, J., Fraga, A. \& Moreno-Pelayo, V. (2008). Hacia una web semántica social. El profesional de la información, 17(1), 78-85.

Moreno Romero, A. (2010). Profesionales con múltiples identidades organizativas en la sociedad en red. Acción psicológica, 7(1), 65-73.

ONTSI (Observatorio Nacional de las Telecomunicaciones y de la SI) (2012). Las

Redes Sociales en Internet. Madrid: ONTSI.

Smith, M. \& Kollock, P. (1999). Communities in Cyberspace. London: Routledge. Sterling, B. (1993). The hacker crackdown. US: Bantam Books.

Turkle, S. (1995). El segundo Yo. México D. F.: Trilla.

Ureña, G. V. \& Valenzuela, J. R. (2011). Contactos de redes sociales en línea como repositorios de información. Revista de Universidad y Sociedad del Conocimiento, RUSC, 8(1), 128-155. 
Wellman, B. (2004). The three ages of Internet studies: ten, five and zero years ago. New Media \& Society, 6(1), 123-129. London: SAGE Publications.

Zenithoptimedia (2010). Móviles y publicidad. Percepciones, usos y tendencias. Madrid: Zenithoptimedia.

Artículo del Grupo de Investigación sobre Periodismo Digital y Banda Ancha (Sistemas de producción del periodismo digital español en el contexto internacional e impacto de la banda ancha) de la Universitat Abat Oliba CEU (UAO-CEU). Financiación: Ayudas a la Investigación 2012 CEU-Banco Santander. Código del proyecto: B112AR08. 